\title{
Energetics of native point defects in GaN: a density-functional study
}

\author{
Giacomo Miceli*, Alfredo Pasquarello \\ Chaire de Simulation à l'Echelle Atomique (CSEA), Ecole Polytechnique Fédérale de Lausanne (EPFL), CH-1015 Lausanne, Switzerland
}

\begin{abstract}
We study the formation energies of native point defects in GaN through density-functional theory. In our first-principles scheme, the band edges are positioned in accord with hybrid density functional calculations, thus yielding a band-gap in agreement with experiment. With respect to previous semilocal calculations, the calculated formation energies and charge transition levels are found to be significantly different in quantitative terms, while the overall qualitative trend remains similar. In Ga-rich conditions, the nitrogen vacancy corresponds to the most stable defect for all Fermi energies in the band gap, but its formation energy is too high to account for autodoping. Our calculations also indicate that the gallium vacancy does not play any compensating role in $n$-type GaN.
\end{abstract}

Keywords: III-nitride, GaN, native defects, hybrid density functional

\section{Introduction}

The discovery of $p$ conductivity in magnesium-doped GaN and the achievement of highly efficient blue-light-emitting diodes [1-3] have made of $\mathrm{GaN}$ one of the most promising 'compounds for future optoelectronic device applications in the visible and ultra-violet frequency range [4]. Furthermore, its wide and direct band gap, high thermal conductivity, favorable electron transport, and large breakdown fields also make this 'compound ideally suited for high-power and high-frequency electronic devices [5]. Despite the rapid commercialization of GaN-based devices, their performance is still not optimal. For instance, while the current $p$-doping level is sufficient for light'emitting diodes, the lack of an efficient $p$-doping is recognized as the bottleneck for obtaining GaN-based laser diodes. Native defects are commonly thought to play a role in the limited hole conductivity of $p$-type GaN. For some time, native defects such as nitrogen vacancies have also been invoked for explaining the $n$-type conductivity of as-grown nonintentionally doped $\mathrm{GaN}$ samples [6, 7]. However, the current interpretation is that this autodoping rather results from extrinsic impurities like oxygen, 'due to the high calculated defect formation energies of vacancy defects [8]. Native defects are also thought to be responsible for shortening the lifetime of electronic devices and for their low performances. In this context, it is clear that the availability of accurate calculations of native defects plays a critical role for further progress.

Several density-functional studies have provided a comprehensive description of the structural and energetic properties of native point defects in GaN [9-12]. However, these studies generally relied on semilocal density functionals which severely underestimate the band gap of GaN. Furthermore, the common

\footnotetext{
${ }^{*}$ Corresponding author

Email address: giacomo.miceli@epfl.ch (Giacomo Miceli)
}

practice of aligning the calculated valence band edge to the experimental one further led to incorrectly positioned defect levels. Early calculations also suffered from small simulation cells and inappropriate treatment of the finite-size effect. Given their potential role in many of the observed electronic phenomena of $\mathrm{GaN}$, it is highly desirable to revisit the formation energies of native defects in $\mathrm{GaN}$ through the use of state-of-the-art methodologies.

In this work, we address the formation energies of native point defects in GaN using a first-principles scheme which reproduces the band gap of $\mathrm{GaN}$ and overcomes the alignment problem. Vacancies, interstitials, and antisites are studied in all their stable charge states. We find significantly different formation energies and defect levels than in previous calculations, but the general qualitative trends are confirmed. In Ga-rich conditions, the nitrogen vacancy is found to be the most stable defect throughout the band gap, but its formation energy is too high to account for autodoping. Our calculated formation energies indicate that the gallium vacancy is unlikely to play any compensating role in $n$-type conditions.

\section{Methods}

In this work, the electronic structure is described through the density functional proposed by Perdew, Becke, and Ernzerhof (PBE) [13]. We use a computational framework based on norm-conserving pseudopotentials and plane-wave basis sets, as implemented in the Quantum-EsPREsso suite of programs [14]. Gallium $3 d$ electrons are explicitly included in the valence shell. The kinetic energy cut-off is set at 75 Ry. Spinunrestricted calculations are performed when unpaired electrons occur. Only the lowest spin multiplicity are considered. All the defect structures are fully relaxed. The Brillouin zone sampling is carried out through the use of an off-center sin- 
gle $k$-point as defined in the Monkhorst-Pack scheme [15], corresponding to the equivalent of the Baldereschi point for an hexagonal lattice [16].

The formation energy of a defect $X$ in the charged state $q$ as a function of the Fermi energy $\epsilon_{\mathrm{F}}$, referenced to the valence band maximum $\epsilon_{\mathrm{v}}$ reads

$$
\begin{aligned}
E_{\mathrm{f}}\left[X^{q}\right]= & E_{\mathrm{tot}}\left[X^{q}\right]-E_{\mathrm{tot}}[\text { bulk }]-\sum_{\alpha} n_{\alpha} \mu_{\alpha}+ \\
& +q\left(\epsilon_{\mathrm{F}}+\epsilon_{\mathrm{V}}\right)+E_{\mathrm{corr}}^{q}
\end{aligned}
$$

where $E_{\text {tot }}\left[X^{q}\right]$ and $E_{\text {tot }}[$ bulk] are the total energies of supercell calculations with and without the defect, respectively. In Eq. (1), $\mu_{\alpha}$ is the chemical potential of the atomic species $\alpha$ and $n_{\alpha}$ the number of atomic species $\alpha$ added $\left(n_{\alpha}>0\right)$ or removed $\left(n_{\alpha}<0\right)$ from the pristine compound. We consider Ga-rich and N-rich conditions, in which $\mu_{\mathrm{Ga}}=\mu_{\mathrm{Ga}}$ [bulk $]$ and $\mu_{\mathrm{N}}=$ $\mu_{\mathrm{N}_{2}}$, respectively. In either of the considered conditions, the chemical potential of the other species is determined according to the thermodynamic equilibrium condition in $\mathrm{GaN}: \mu_{\mathrm{GaN}}=$ $\mu_{\mathrm{Ga}}+\mu_{\mathrm{N}} . E_{\mathrm{corr}}^{q}$ accounts for finite-size corrections and includes a potential alignment term [17, 18].

To overcome the band-gap underestimation problem of semilocal functionals, we use the band-edge positions as found with the Heyd-Scuseria-Ernzerhof hybrid density functional (HSE) [19-21]. We fixed the fraction of Fock exchange to $\alpha=0.31$ in order to reproduce the experimental band gap of GaN. Through an alignment based on the average electrostatic potential, the band-edge positions achieved with the HSE functional are then positioned with respect to the electronic structure achieved in the PBE [22, 23]. The validity of this alignment scheme rests on the fact that energy levels of atomically localized defects do not vary when going from the PBE to the hybridfunctional description [21, 24-26]. For defect states involving anionic $2 p$ orbitals, self-interaction effects leading to charge localization are better described in hybrid-functional approaches [27, 28]. However, these effects generally do not affect the defect formation energy in a significicant way [29].

\section{Results}

All the defects are modeled starting from a 108-atom supercell of wurtzite GaN subject to periodic boundary conditions. The larger simulation cell used here allows for a better description of the atomic relaxation around the defects compared to earlier studies [9, 10]. The calculated formation energies of all native defects in $\mathrm{GaN}$ are shown as a function of the Fermi energy in Fig. 1, for both Ga-rich and N-rich conditions. The formation energies achieved for the Fermi energy at the top of the valence band are reported in Table 1 The corresponding charge transition levels are summarized in Table 2. Compared to previous semilocal calculations [10, 12, 30], we notice significant quantitative differences reaching up to $2 \mathrm{eV}$ for formation energies and up to $1 \mathrm{eV}$ for charge transition levels. Nevertheless, the overall qualitative trends reported in Refs. [12, 30] can still be recognized. Variations can be attributed to various effects, including the size of the simulation cell, the k-point sampling,
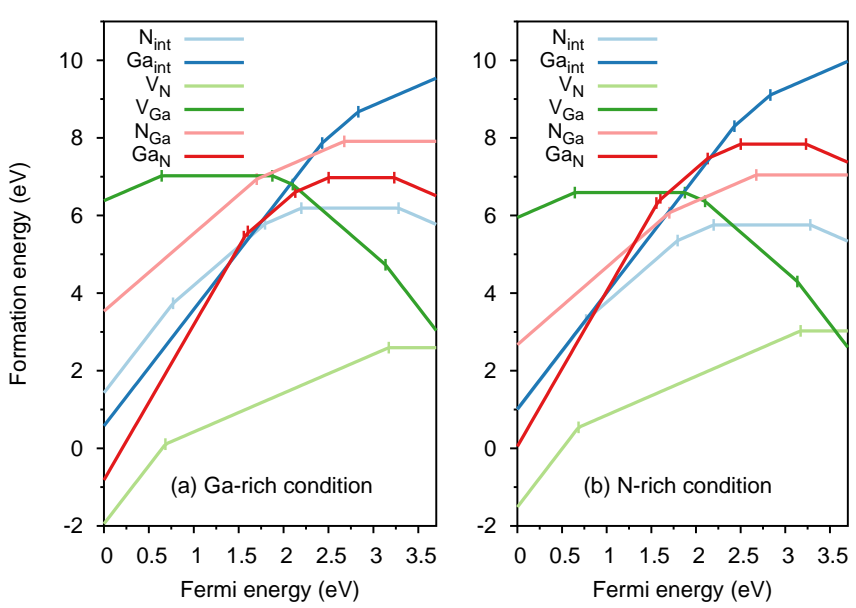

Figure 1: Calculated formation energies of native point defects in $\mathrm{GaN}$ as a function of Fermi energy. In panels (a) and (b), Ga-rich and N-rich conditions are assumed, respectively. For each defect, only the lowest formation energy is shown for varying Fermi energy. The kinks in the solid line are marked by vertical bars and correspond to thermodynamic charge transition levels.

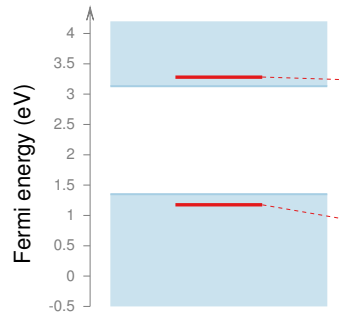

(a)

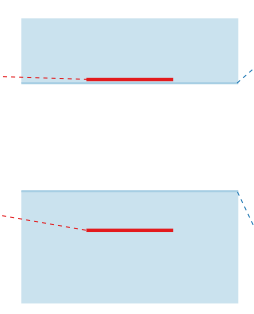

(b)

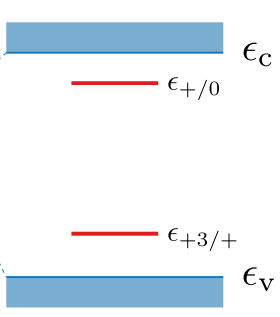

(c)
Figure 2: Charge transition levels of the nitrogen vacancy calculated with the PBE functional (a) without and (b) with finite-size corrections. In panel (c) the correct band gap is obtained through the use of the HSE hybrid functional. The defect levels calculated at the PBE level are positioned in the HSE band-gap by aligning the electronic structures through the average electrostatic potentials.

the treatment of Ga $3 d$ states, the finite-size corrections, and the alignment procedure.

It should be stressed that a pure semilocal calculation may yield quantitatively and qualitatively erroneous results. To illustrate the shifts undergone by the defect energy level upon the variation of the adopted level of theory, we focus on the nitrogen vacancy defect. This defect shows two charge transition levels in the band gap, $\epsilon_{+3 /+}$ and $\epsilon_{+/ 0}$, as shown in Fig. 1. In a pure PBE calculation, the calculated band gap of 1.78 $\mathrm{eV}$ severely underestimates the experimental one (3.5 eV, Ref. [31]) and neither of the two charge transition levels are found in the band gap, as shown in Fig.2]a). We can nevertheless obtain the position of the defect levels by sampling the Brillouin zone through a single off-center k-point. In such a setup, the energy gap in the calculation increases to $3.95 \mathrm{eV}$, the defect charge distributions are atomically well localized, and the two charge transition levels can meaningfully be determined. The obtained defect energy levels can then be positioned with respect to the actual $\mathrm{GaN}$ band edges obtained from a bulk calculation with a dense k-point sampling through the alignment of the average electrostatic potential. Next, finite-size effects need to be con- 
Table 1: Formation energies of native point defects in $\mathrm{GaN}$ calculated for the Fermi level at the top of the valence band in Ga-rich and N-rich conditions.

\begin{tabular}{|c|c|c|c|}
\hline \multirow[b]{2}{*}{ Defect } & \multirow[b]{2}{*}{ Charge state } & \multicolumn{2}{|c|}{$\overline{E_{f}(e V)}$} \\
\hline & & Ga-rich & N-rich \\
\hline \multirow{5}{*}{$\mathrm{N}_{\text {int }}$} & +3 & 1.43 & 0.99 \\
\hline & +2 & 2.20 & 1.76 \\
\hline & +1 & 4.00 & 3.56 \\
\hline & 0 & 6.19 & 5.75 \\
\hline & -1 & 9.47 & 9.03 \\
\hline \multirow[t]{3}{*}{$\mathrm{Ga}_{\text {int }}$} & +3 & 0.58 & 1.02 \\
\hline & +2 & 3.01 & 3.45 \\
\hline & +1 & 5.84 & 6.28 \\
\hline \multirow[t]{3}{*}{$\mathrm{V}_{\mathrm{N}}$} & +3 & -1.95 & -1.51 \\
\hline & +1 & -0.58 & -0.14 \\
\hline & 0 & 2.59 & 3.03 \\
\hline \multirow[t]{4}{*}{$\mathrm{V}_{\mathrm{Ga}}$} & 0 & 7.02 & 6.58 \\
\hline & -1 & 8.90 & 8.46 \\
\hline & -2 & 11.00 & 10.56 \\
\hline & -3 & 14.14 & 13.70 \\
\hline \multirow[t]{3}{*}{$\mathrm{N}_{\mathrm{Ga}}$} & +2 & 3.53 & 2.66 \\
\hline & +1 & 5.23 & 4.36 \\
\hline & 0 & 7.91 & 7.04 \\
\hline \multirow[t]{6}{*}{$\mathrm{Ga}_{\mathrm{N}}$} & +4 & -0.82 & 0.05 \\
\hline & +3 & 0.79 & 1.66 \\
\hline & +2 & 2.34 & 3.21 \\
\hline & +1 & 4.47 & 5.34 \\
\hline & 0 & 6.97 & 7.84 \\
\hline & -1 & 10.21 & 11.08 \\
\hline
\end{tabular}

Table 2: Calculated charge transition levels $\epsilon_{q / q^{\prime}}$ between the charges states $q$ and $q^{\prime}$ for native point defects in GaN. The defect levels are referred to the top of the valence band.

\begin{tabular}{|c|c|c|}
\hline$\overline{\text { Defect }}$ & $q / q^{\prime}$ & $\overline{\epsilon_{q / q^{\prime}}(\mathrm{eV})}$ \\
\hline \multirow[t]{4}{*}{$\mathrm{N}_{\text {int }}$} & $+3 /+2$ & 0.77 \\
\hline & $+2 /+1$ & 1.80 \\
\hline & $+1 / 0$ & 2.20 \\
\hline & $0 /-1$ & 3.28 \\
\hline \multirow[t]{2}{*}{$\mathrm{Ga}_{\text {int }}$} & $+3 /+2$ & 2.43 \\
\hline & $+2 /+1$ & 2.83 \\
\hline \multirow[t]{2}{*}{$\mathrm{V}_{\mathrm{N}}$} & $+2 /+1$ & 0.68 \\
\hline & $+1 / 0$ & 3.17 \\
\hline \multirow[t]{3}{*}{$\mathrm{V}_{\mathrm{Ga}}$} & $0 /-1$ & 1.88 \\
\hline & $-1 /-2$ & 2.10 \\
\hline & $-2 /-3$ & 3.13 \\
\hline \multirow[t]{2}{*}{$\mathrm{N}_{\mathrm{Ga}}$} & $+2 /+1$ & 1.70 \\
\hline & $+1 / 0$ & 2.67 \\
\hline \multirow[t]{5}{*}{$\mathrm{Ga}_{\mathrm{N}}$} & $+4 /+3$ & 1.56 \\
\hline & $+3 /+2$ & 1.60 \\
\hline & $+2 /+1$ & 2.13 \\
\hline & $+1 / 0$ & 2.50 \\
\hline & $0 /-1$ & 3.23 \\
\hline
\end{tabular}

sidered as they are still significant for a simulation cell of 108 atoms. For instance, for the nitrogen vacancy in its +3 charge state, the correction term due to spurious electrostatic interactions is as large as $1.04 \mathrm{eV}$. For the $\mathrm{Ga}_{\mathrm{N}}$ defect in its +4 charge state, the finite-size correction reaches $2.12 \mathrm{eV}$, due to the high nominal charge of the defect. The effect of the finite-size corrections on the charge transition levels of the nitrogen vacancy are illustrated in Fig. 2(b). In the final step of our procedure, the band edges obtained with the HSE functional are positioned on the same energy scale as the defect levels obtained with the PBE functional. This is achieved through the alignment of the bulk electrostatic potentials in the HSE and PBE calculations [21, 24-26]. As shown in Fig. 2] (c), this last step produces the opening of the band gap which thereby encloses the two charge transition levels of the nitrogen vacancy.

The validity of our methodological scheme can further be assessed by comparing our results for the nitrogen vacancy with those obtained within a fully consistent hybrid functional approach [32]. The $+3 /+$ and $+/ 0$ charge transition levels found in the present work at 0.68 and $3.17 \mathrm{eV}$ compare very well with the respective hybrid-functional levels at 0.47 and $3.25 \mathrm{eV}$ [32]. Similarly, the formation energy of the neutral state found at 2.6 $\mathrm{eV}$ in our calculation (cf. Ga-rich conditions in Fig. 1) shows a fair agreement with the corresponding hybrid functional result of $3.1 \mathrm{eV}$ [32]. The quality of this agreement provides confidence in the accuracy of our calculation scheme. Remarkably, this level of accuracy is presently achieved with PBE calcula- 
tions which imply a significantly lower computational cost than fully consistent hybrid functional calculations, thereby enabling us to comprehensively address the energetics of native defects in $\mathrm{GaN}$.

In Ga-rich conditions, the nitrogen vacancy is found to be the most stable defect for all Fermi energies in the band gap. In particular, this condition also applies for Fermi energies close to the conduction band, unlike previous calculations which found the gallium vacancy at lower energies [12]. In $p$-type conditions, the calculated formation energy of the nitrogen vacancy is $-2.0 \mathrm{eV}$, indicating that such defects would lead to strong compensation effects in this regime. In $n$-type conditions, the formation energy is $2.6 \mathrm{eV}$, lower by more than $1 \mathrm{eV}$ with respect to previous semilocal calculations [12], but still too high to assign a role to this defect in the observed $n$-type conductivity of nonintentionally doped GaN. Indeed, the calculated formation energy of the nitrogen vacancy in its charge state +1 results in an $n$-type carrier concentration of $\sim 10^{11} \mathrm{~cm}^{-3}$ at typical growth temperatures around $1050^{\circ} \mathrm{C}$ [7]. This level of autodoping is orders of magnitude lower than the lowest $n$-type carrier concentration measured in nominally undoped samples, which ranges from $10^{17}$ to $10^{19} \mathrm{~cm}^{-3}$ [7]. This further supports the conclusion drawn from previous calculations that the observed $n$-type carriers do not result from intrinsic defects such as nitrogen vacancies [9, 10].

In N-rich conditions, the nitrogen vacancy is still the lowest energy defect for all Fermi energy in the band gap but a small energy range in the vicinity of the conduction band, where the gallium vacancy in its -3 charge state shows slightly lower formation energies. However, the calculated formation energies remain too high for the gallium vacancy playing any relevant compensating role in $n$-type conditions.

\section{Conclusion}

We provide a comprehensive and accurate description of formation energies of native point defects in GaN using a stateof-the-art first-principles methodology. Such a description is fundamental for the interpretation of the electronic properties of $\mathrm{GaN}$ related to such defects.

\section{Acknowledgements}

Financial support is acknowledged from the Swiss National Science Foundation (Grants Nos. 200020-152799 and 200020134600). We used computational resources of CSCS and CSEA.

\section{References}

[1] H. Amano, M. Kito, K. Hiramatsu, I. Akasaki, Jpn. J. Appl. Phys. 28 (1989) L2112.

[2] S. Nakamura, T. Mukai, M. Senoh, N. Iwasa, Jpn. J. Appl. Phys. 31 (1992) L139.

[3] S. Nakamura, T. Mukai, M. Senoh, Appl. Phys. Lett. 64 (1994) 16871689.

[4] S. Nakamura, M. R. Krames, Proc. IEEE 101 (2013) 2211-2220.

[5] S. Pearton, J. Zolper, R. Shul, F. Ren, J. Appl. Phys. 86 (1999) 1-78.
[6] H. P. Maruska, J. Tietjen, Appl. Phys. Lett. 15 (1969) 327-329.

[7] M. Ilegems, H. C. Montgomery, J. Phys. Chem. Solids 34 (1973) 885.

[8] C. G. Van de Walle, J. Neugebauer, J. Appl. Phys. 95 (2004) 3851-3879.

[9] J. Neugebauer, C. G. Van de Walle, Phys. Rev. B 50 (1994) 8067.

[10] P. Bogusławski, E. L. Briggs, J. Bernholc, Phys. Rev. B 51 (1995) 17255.

[11] J. Neugebauer, C. G. Van de Walle, in: Mater. Res. Soc. Symp. Proc., volume 395, Cambridge Univ Press, p. 645.

[12] S. Limpijumnong, C. G. Van de Walle, Phys. Rev. B 69 (2004) 035207.

[13] J. P. Perdew, K. Burke, M. Ernzerhof, Phys. Rev. Lett. 77 (1996) 38653868.

[14] Giannozzi P. et al., J. Phys.: Condens. Matter 21 (2009) 395502.

[15] H. J. Monkhorst, J. D. Pack, Phys. Rev. B 13 (1976) 5188.

[16] A. Baldereschi, Phys. Rev. B 7 (1973) 5212-5215.

[17] C. Freysoldt, J. Neugebauer, C. Van de Walle, Phys. Rev. Lett. 102 (2009) 016402.

[18] H.-P. Komsa, T. T. Rantala, A. Pasquarello, Phys. Rev. B 86 (2012) 045112.

[19] J. Heyd, G. E. Scuseria, M. Ernzerhof, J. Chem. Phys. 118 (2003) 82078215 .

[20] J. Heyd, G. E. Scuseria, M. Ernzerhof, J. Chem. Phys. 124 (2006) 219906.

[21] H.-P. Komsa, P. Broqvist, A. Pasquarello, Phys. Rev. B 81 (2010) 205118.

[22] P. Dahinden, P. Broqvist, A. Pasquarello, Phys. Rev. B 81 (2010) 085331.

[23] G. Miceli, A. Pasquarello, Microelectron. Eng. 109 (2013) 60-63.

[24] A. Alkauskas, P. Broqvist, A. Pasquarello, Phys. Rev. Lett. 101 (2008) 046405.

[25] A. Alkauskas, P. Broqvist, A. Pasquarello, Phys. Status Solidi B 248 (2011) 775-789.

[26] A. Alkauskas, A. Pasquarello, Phys. Rev. B 84 (2011) 125206.

[27] G. Pacchioni, F. Frigoli, D. Ricci, J. A. Weil, Phys. Rev. B 63 (2000) 054102.

[28] J. Lægsgaard, K. Stokbro, Phys. Rev. Lett. 86 (2001) 2834-2837.

[29] A. Carvalho, A. Alkauskas, A. Pasquarello, A. K. Tagantsev, N. Setter, Phys. Rev. B 80 (2009) 195205.

[30] C. G. Van de Walle, J. Neugebauer, J. Appl. Phys. 95 (2004) 3851-3879.

[31] O. Madelung, Semiconductors: Data Handbook, Springer, 2004.

[32] Q. Yan, A. Janotti, M. Scheffler, C. G. Van de Walle, Appl. Phys. Lett. 100 (2012) 142110 